\title{
Modeling the Strategic Behavior of the Iberian Electricity Market Producers using Time Series Analysis
}

\author{
Ricardo Faria \\ ISEL \\ Lisbon, Portugal \\ rfaria_88@hotmail.com
}

\author{
Jorge Sousa, MIEEE \\ ISEL \\ INESC-ID \\ Lisbon, Portugal \\ jsousa@deea.isel.ipl.pt
}

\author{
Ana Martins \\ ISEL \\ BRU-UNIDE \\ Lisbon, Portugal \\ anamartins@adm.isel.pt
}

\author{
João Lagarto \\ ISEL \\ INESC-ID \\ MIT-Portugal \\ Lisbon, Portugal \\ jlagarto@deea.isel.ipl.pt
}

\begin{abstract}
The Iberian Electricity Market (MIBEL) emerges in the context of the integration and cooperation between the Portuguese and Spanish electricity markets, in response to the European Union incentive for regional electricity markets creation. The present study, focus on the modeling and forecasting of the hourly competitive strategies of the electricity producers in the MIBEL. For this analysis, the studied variable was the MIBEL's conjectural variation, which estimates the level of competitiveness of the electricity producers on the day-ahead electricity market. The methodology adopted for forecasting was time series analysis, using ARIMA and exponential smoothing models. The results obtained show that the estimated models that best suit the hourly MIBEL conjectural variation forecast were mainly of the ARIMA seasonal type with daily seasonality, followed by ARIMA non-seasonal type models. It was also observed, that the selected models were mainly estimated with a time series of 5 working days.
\end{abstract}

Index Terms-ARIMA models, Conjectural variations, MIBEL, Strategic behavior, Time series

\section{INTRODUCTION}

Electricity markets are recent entities arising from the liberalization process of the electricity industry that has been undergoing in many countries. Due to the particular nature of electricity of which it can be emphasized the fact that: it cannot be stored in an economic way; supply must meet demand at all moments and the inelastic nature of the demand, electricity markets are extremely complex and pose new difficulties to the market players. Despite this complexity, the liberalization process has been consistently established throughout the world leading even to the integration of electricity markets, of which it can be referred as noticeable examples the cases of the Nord Pool, the Pennsylvania - New Jersey - Maryland Interconnection (PJM) and the Iberian electricity market (MIBEL).

Among the difficulties facing market players, the volatility and uncertainty of the electricity market price is one of them. In particular, for the electricity producers aiming at profit maximization, the modeling and forecasting of the electricity market price is of paramount importance.

The commonly used methods to model and forecast the electricity prices can be divided into two distinct groups:

This work was supported by national funds through FCT - Fundação para a Ciência e a Tecnologia, under project PEst-OE/EEI/UI4064/2011. statistical models and non-statistical models [1]. Statistical models are separated in three categories, models based in time series analysis, econometric models and the artificial intelligence approach. Non-statistical models are composed by models that use simulation and equilibrium models.

Models based on time series analysis focus on the explanation of a given variable according to time and its past occurrences. Examples are the application of Auto-Regressive (AR) models [2], Auto-Regressive Integrated Moving Average (ARIMA) models [3] and ARIMA models with a wavelet filter [4] to forecast the Spanish and Californian electricity market prices.

Econometric models take advantage of the possibility of complex and dynamic transactions and applications on financial studies. The application of such models focuses generally on the electricity price forecast such as in [5] and [6].

Models based on artificial intelligence are mainly composed by artificial neural networks (ANN) models, fuzzy logic models and even the combination of ANN with fuzzy logic. Examples are the implementation of multilayer perceptron ANN models [7] and the Adaptative Neuro-Fuzzy Inference System (ANFIS) [8].

Within non-statistical models, equilibrium analysis models using artificial agents are used in [9], and as an example of work that uses simulation models for price forecast is [10].

With the creation of electricity markets their performance was also subject of study and analysis. In [11] a study of the Portuguese and Spanish electricity markets integration is conducted through equilibrium model analysis to describe the strategic behavior of the market participants. In this study a conjectural variations model is developed and a parameter called conjectural variation is obtained. This parameter reflects the level of competitiveness of the electricity producers on the market.

The development of the study performed in [11] is carried out in [12]. This study besides, accomplishing the creation of the simulator SiMEC v3.0 based on a conjectural variations model, analyzes the impact of the creation of MIBEL in the level of competitiveness of the electricity producers. The developed simulator contains two modes of operation, the estimation mode and the simulation mode. The estimation mode uses as inputs the $\mathrm{CO}_{2}$ emission prices, fuel data, data 
from power plants and day-ahead electricity market data. In this mode of operation SiMEC v3.0 computes the conjectural variation value. The simulation mode allows computing the day-ahead electricity market price using as input the conjectural variation value.

The present study draws on the conjectural variation model used in [11] and the simulator developed in [12], in order to model and forecast the hourly strategic behavior of MIBEL electricity producers for 5 working days, using time series analysis through the study of the conjectural variation parameter.

This paper is structured as follows. The methodology used to model and forecast the MIBEL conjectural variation is presented in section II. In section III the modeling results are presented. In section IV the forecasting results are shown. The conclusions drawn are presented in section $\mathrm{V}$.

\section{Methodology And Time Series Models USED}

The purpose of this paper, as mentioned before, is to model the strategic behavior of the electricity producers in the MIBEL for working days using time series analysis. For this purpose, the modeling and forecasting of the MIBEL hourly conjectural variation was performed. The hourly conjectural variation represents de degree of competitiveness presented by the electricity producers acting on the MIBEL day-ahead market.

According to [11], a conjectural variation value equal to -1 means that the perfectly competitive outcome is obtained. A conjectural variation equal to 0 represents the Cournot competitive outcome. Collusive behaviors are present when the conjectural variation assumes values greater than 0 .

The chronological series of the conjectural variation data was obtained through the simulator SiMEC v3.0 in estimation mode. In the MIBEL, to allocate interconnection capacity between the Portuguese and Spanish power networks in the day-ahead and intraday markets the market splitting mechanism is used causing different prices for both markets.

To overcome this problem, the MIBEL day-head market electricity price considered was a weighted average price, given by:

$$
\mathrm{P}_{\text {MIBEL }}=\frac{\mathrm{P}_{\mathrm{ES}} \cdot \mathrm{E}_{\mathrm{ES}}+\mathrm{P}_{\mathrm{PT}} \cdot \mathrm{E}_{\mathrm{PT}}}{\mathrm{E}_{\mathrm{ES}}+\mathrm{E}_{\mathrm{PT}}}
$$

Where:

$\mathrm{P}_{\text {MIBEL }}$ is the MIBEL day-ahead market price (€/MWh);

$\mathrm{P}_{\mathrm{ES}}$ is the Spanish electricity market price (€/MWh);

$\mathrm{E}_{\mathrm{ES}}$ is the energy traded in the Spanish electricity market (MWh);

$\mathrm{P}_{\mathrm{PT}}$ is the Portuguese electricity market price ( $\left.€ / \mathrm{MWh}\right)$;

$\mathrm{E}_{\mathrm{PT}}$ is the energy traded in the Portuguese electricity market (MWh).

In order to model and forecast the MIBEL hourly conjectural variation for working days, a methodology was developed using programming in Microsoft Office 2007 Visual Basic for Applications (VBA) and in Statistical Package for the Social Sciences (SPSS), version IBM SPSS Statistics 19.
The developed methodology performs the hourly forecast for a window of 5 working days, requiring hourly conjectural variation data of 30 working days. The methodological approach is as follows:

1. Estimation of 12 models and respective 5 working days forecasting;

2. Selection of the model and respective forecast that simultaneously validates the model requirements and contains the lowest Normalized Bayesian Information Criterion (BIC) value.

Using the Expert Modeler tool present in the SPSS software 12 models were estimated. This algorithm identifies and estimates an appropriate seasonal or non-seasonal ARIMA or exponential smoothing model for each time series. The 12 models were obtained using 6 alternative time series lengths $(5,10,15,20,25$ and 30 working days) and by considering or not the outliers present in the data for the estimation process [13].

The selection of the better model and forecasting between the 12 estimated models is performed in order to obtain the one which simultaneously validates the model requirements and that contains the lowest BIC value presented in the estimation results.

\section{MODEL ESTIMATION}

In order to model the strategic behavior of the electricity producers in the MIBEL day-ahead market, the study of 30 time windows using the process described above was performed. For this study, hourly conjectural variation data between January 25 and October 30 of 2011 was used.

Through the analysis of the conjectural variation data, the presence of any cyclic component or global tendency in the time series was not verified. By this, it was not observed any oscillatory motions in medium term or even overall evolution of increase or decrease of the time series. However, it was possible to verify the existence of additive seasonality with daily frequency.

The modeling results obtained show that the models selected were not the same, varying depending on each studied time window. It was observed that 27 of the selected models were of the seasonal ARIMA type with daily seasonality. The remaining 3 selected models were of non-seasonal ARIMA type.

By the analysis of the modeling results of the different time windows, it was possible to observe that as the size of the time series used for estimation of the different models increases, there is a decrease of the quality of adjustment. In this way, recent data of conjectural variation leads usually to good modeling results in order to forecast 5 working days.

This study reveals that for the 30 time windows addressed, mostly of the models selected were estimated from a time series with 5 working days (40\%) and subsequently with 10 working days (20\%). However, for time series with 15, 20, 25 and 30 working days the number of models selected were the same $(10 \%$ each). According to the size of the time series used to estimate, the number of selected models can be observed in Fig. 1. 


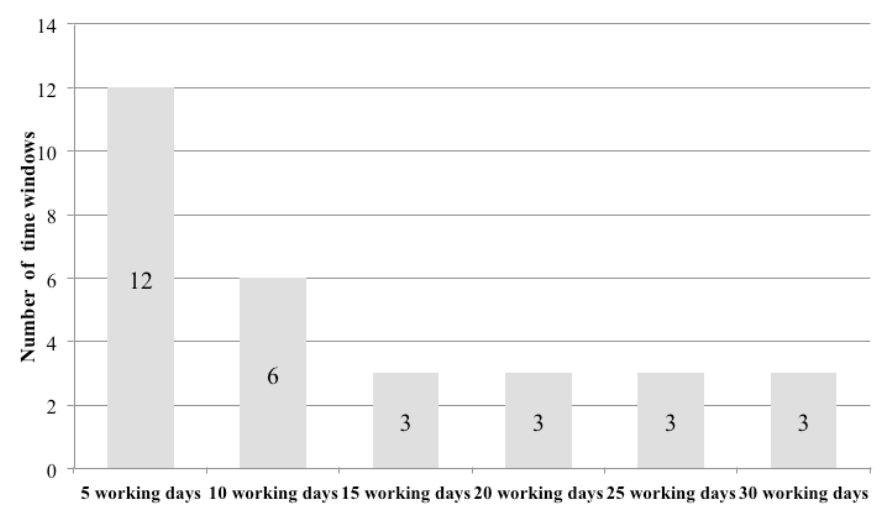

Figure 1. Number of selected models according to the size of the time series used for model estimation

The BIC value obtained by the selected models for each time window is shown in Fig. 2.

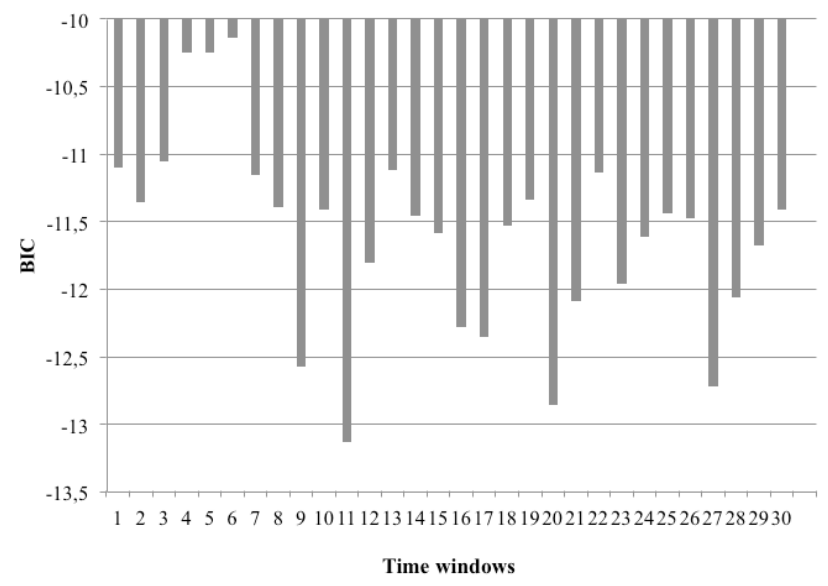

Figure 2. BIC of the model selected for each time window.

Accessing the quality of adjustment in terms of $\mathrm{R}^{2}$ for the different selected models, it was obtained an average $\mathrm{R}^{2}$ value of 0.975 , with a maximum of 0.998 and a minimum of 0.806 . Therefore, on average the $\mathrm{R}^{2}$ value obtained for the study presented is very good, showing a good fit between the values of the conjectural variation estimated and the conjectural variation time series data for the time windows analyzed. The $\mathrm{R}^{2}$ obtained for each time window is presented in Fig. 3 .

Through the different models selected, it was possible to conclude that in order to forecast a certain hour it was needed conjectural variation data in a maximum of 3 working days before. Namely, $20 \%$ of the time windows required conjectural variation data of 1 working day before, $53 \%$ required 2 working days before and $27 \%$ required 3 working days before in order to forecast a certain hour. Thus, it can be noted that the competitive level recorded by the electricity producers in MIBEL day-ahead market at a certain hour was mostly explainable through the 2 working days before that hour.

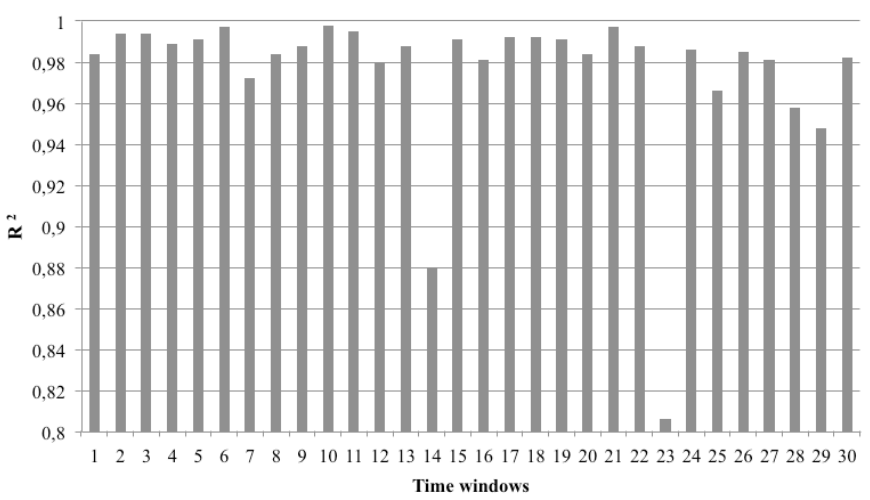

Figure 3. $\mathrm{R}^{2}$ of the model selected for each time window.

\section{FORECASTING RESULTS}

To analyze the accuracy of the conjectural variation forecasting results, the study of the mean absolute error for a forecast window of 1 and 5 working days was performed using the selected model. Through the results obtained, it was possible to verify that the mean absolute error of the forecasting results with 1 working day window usually had a lower value compared to results obtained with 5 working days. The mean absolute error for a forecast of 1 and 5 working days for each time window is presented in Fig. 4.

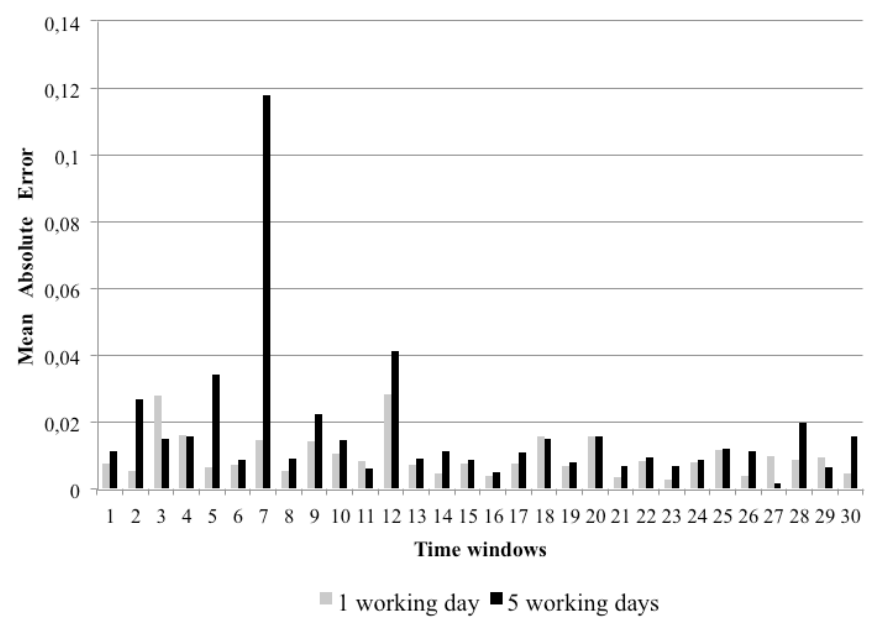

Figure 4. Mean absolute error for a 1 and 5 working days forecast window.

In order to analyze the forecasting results obtained for the hourly strategic behavior of the electricity producers, three time windows will be detailed in Table I.

For an overview of the selected models performance in terms of estimation, forecasting results and the strategic behavior shown by the electricity producers in the MIBEL dayahead market, the graphical analysis of the conjectural variation values calculated by the simulator SiMEC v3.0 in comparison with fitted/forecasted conjectural variation values was performed. Through this study it was observed that the selected models presented difficulty in forecasting the off-peak hours, mainly when lower electricity day-ahead market price occurred leading to a sharp decrease of the conjectural variation value (sharp increase of the competitive level). 
TABLE I. MODELING DESCRIPTION FOR THREE TIME WINDOWS

\begin{tabular}{c|c|c|c|c}
$\begin{array}{c}\text { Forecasting } \\
\text { window }\end{array}$ & $\begin{array}{c}\text { Time series } \\
\text { size window } \\
\text { in working } \\
\text { days }\end{array}$ & $\mathbf{R}^{2}$ & BIC & Model selected \\
\hline $\begin{array}{c}\text { March17 to } \\
\text { March 23 }\end{array}$ & 5 & 0.994 & -11.354 & $\operatorname{ARIMA}(1,1,0)(0,1,0)$ \\
\hline $\begin{array}{c}\text { July15 to July } \\
21\end{array}$ & 10 & 0.992 & -12.352 & $\operatorname{ARIMA}(1,0,0)(0,1,1)$ \\
\hline $\begin{array}{c}\text { October11 to } \\
\text { October 17 }\end{array}$ & 15 & 0.986 & -11.572 & $\operatorname{ARIMA}(2,1,2)(1,0,1)$
\end{tabular}

The calculated and fitted/forecasted for the same three time windows are presented in Fig. 5 to 7.

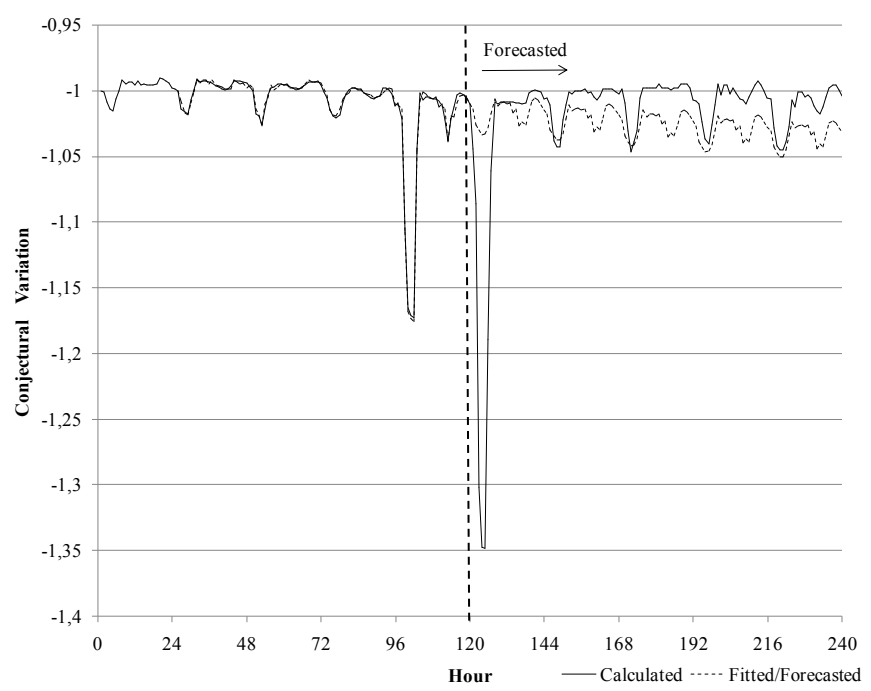

Figure 5. Calculated, fitted and forecasted conjectural variation for the forecasting window of March 17 to March 23 of 2011

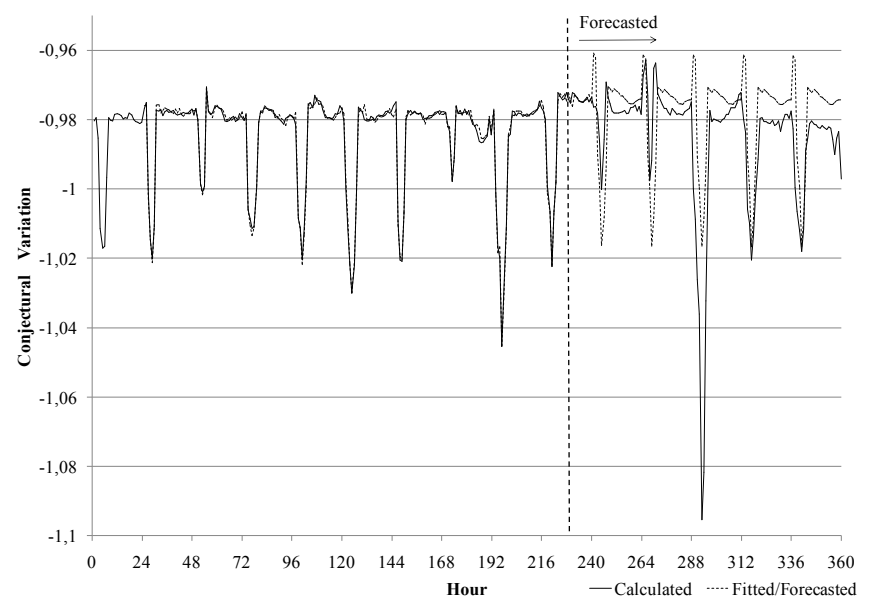

Figure 6. Calculated, fitted and forecasted conjectural variation for the forecasting window of July 15 to July 21 of 2011

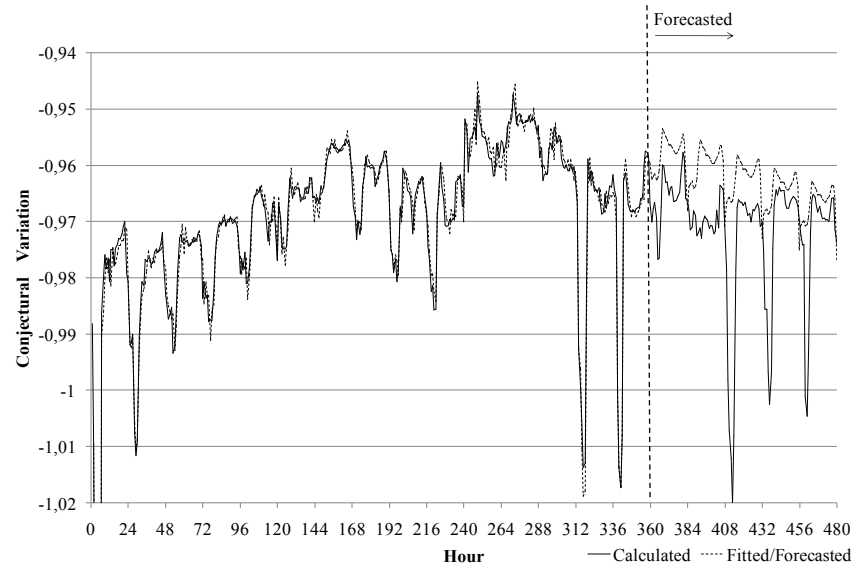

Figure 7. Calculated, fitted and forecasted conjectural variation for the forecasting window of October 11 to October 17 of 2011

In addition, the usefulness of the conjectural variation forecasting results to compute the corresponding market price was analyzed. Using the simulator SiMEC v3.0 in simulation mode the market price was computed (indirect forecast) and the results were compared with the market price forecasting through the time series analysis (direct forecast).

By using t-student tests and Wilcoxon tests it was possible to verify that for a level of significance of $1 \%$ the forecasting results obtained by both methods, presented equal mean absolute errors for a window of 1 and 5 working days. Thus, it can be concluded that the conjectural variation forecasting is useful to predict the corresponding hourly MIBEL day-ahead market price.

The electricity price observed, the direct forecast and the indirect forecast for the forecasting window of 8 of July to 14 of July is presented in Fig. 8.

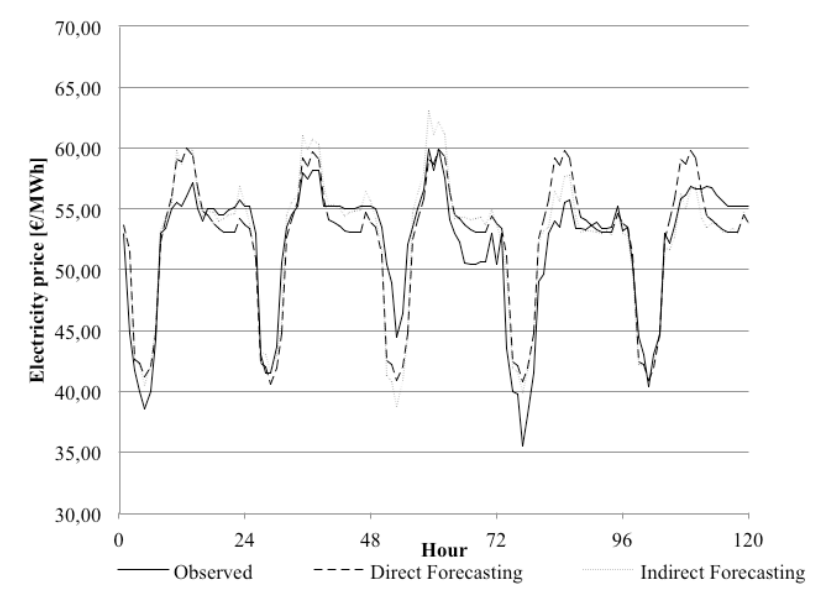

Figure 8. Observed, direct forecast and indirect forecast electricity price for the forecasting window of July 8 to July 14 of 2011 


\section{CONCLUSIONS}

This paper focused on the modeling of the strategic behavior of the electricity producers in MIBEL day-ahead market based on time series analysis, using as variable in study the MIBEL conjectural variation obtained by solving a conjectural variations model.

By studying 30 different time windows, all the selected models were of ARIMA type estimated by performing the detection and treatment of the outliers present in the time series, being 27 of seasonal type and 3 of non-seasonal type.

For the studied time windows, it was observed that as the size of the time series used to estimate the model increased, there was a decrease of the quality of adjustment presenting generally worse modeling results in comparison with smaller sized time series. Thus, using a time series of 5 working days conducted, generally, to better results.

Modeling results show an average $\mathrm{R}^{2}$ value of 0.975 , with a minimum of 0.806 and a maximum of 0.998 . In this way, it can be concluded that the methodology used to model the hourly strategic behavior of the electricity producers in MIBEL day-ahead market presented good fitting results.

After the analysis of different time windows the existence of high volatility in the conjectural variation behavior was verified, following the behavior of the correspondent market price. For this reason, the forecasting results of conjectural variation had difficulty in following the values calculated in the off-peak hours, especially during the occurrence of a sharp decrease on the conjectural variation value.

For the forecasting of a single hour of conjectural variation it was observed that it were necessary hourly conjectural variations data mainly corresponding to the exact 2 days before (in maximum 3 working days before).

In terms of mean absolute error, the forecasting of 1 working day presented better values in comparison with the forecasting of 5 working days. Thus, in order to obtain more accurate results, a 1 day forecasting window should be used.

It was concluded that the direct forecast of market price through time series analysis presented equal mean absolute errors for a window of 1 and 5 working days in comparison with the indirect market price forecasting through the forecasted conjectural variation results obtained. Thereby, it can be considered that the conjectural variation forecasting is useful to forecast the hourly MIBEL day-ahead market price.
A future development of this work will be the modeling and forecasting of the MIBEL day-ahead market conjectural variation for weekends and holidays.

\section{REFERENCES}

[1] G. Li, L. Chen, J. Lawarree, M. Gallanti and A. Venturini, "State of - the - Art of Electricity Price Forecasting," in Proc. 2005 CIGRE/IEEE PES, International Symposium, pp. 110-119.

[2] E. Ni and. P. B. Luh, "Forecasting Power Market Clearing Price and its Discrete PDF Using a Bayesian-based Classification Method," in Proc. 2001 IEEE Power Engenieering Society Winter Meeting, Vol. 3, pp. 1518-1523.

[3] J. Contreras, R. Espinola, F. J. Nogales and A. J. Conejo, “ARIMA Models To Predict Next-Day Electricity Prices," IEEE Transactions on Power Systems, Vol. 18, pp. 1014-1020, Aug. 2003.

[4] A. J. Conejo, M. A. Plazas, R. Espinola and A. B. Molina, "Dayahead Electricity Price Forecasting Using the Wavelet Transform and ARIMA Models," IEEE Transactions on Power Systems, Vol. 20, pp. 1035 -1042, May 2005.

[5] R. Etheir and T. Mount, "Estimating the Volatility of Spot Prices in Restructured Electricity Markets and the Implications for Option Values," unpublished, Dec. 1998. [Online]. Available: http://www.pserc.wisc.edu/documents/publications/papers/1998_gen eral_publications/pserc_31.pdf.

[6] S. Deng, "Pricing Electricity Derivates under Alternative Stochastic Spot Price Models," in Proc. 2000 33rd Hawaii International Conference on System Sciences.

[7] B. R. Szkuta, L. A. Sanavria and T. S. Dillon, "Electricity Price Short-Term Forecasting Using Artificial Neural Networks," IEEE Transactions on Power Systems, Vol. 14, pp. 851-857, Aug. 1999.

[8] C. P. Rodriguez and G. J. Anders, "Energy Price Forecasting in the Ontario Competitive Power System Market," IEEE Transactions on Power Systems, Vol. 19, pp. 366 - 374, Feb. 2004.

[9] D. W. Bunn, "Forecasting Loads and Prices in Competitive Power Markets," in Proc. of the IEEE, pp. 163-169, 2000.

[10] J. Bastian, J. Zhu, V. Banunarayanan and R. Mukerji, "Forecasting Energy Prices in a Competitive Market," IEEE Computer Applications in Power, Vol. 12, pp. 40-45, Jul. 1999.

[11] J. Sousa, "Integration of liberalized electricity energy markets with applications to the MIBEL," PhD. Dissertation, Economics Department, New University of Lisbon, Lisbon, 2005.

[12] J. Lagarto, J. Sousa and A. Martins, "The Impact of The Iberian Electricity Market on the Competitive Behavior of Generating Companies using a Conjectural Variations Approach," in Proc. 2010 7th International Conference on the European Energy Market, pp. 1-9.

[13] D. Pena, G. C. Tiao, and R. S. Tsay. A course in time series analysis. NewYork: John Wiley and Sons, 2001. 